\title{
MODELO DE GESTIÓN DE RIESGOS PARA MEJORAR LA EJECUCIÓN DE OBRAS DE SANEAMIENTO EN LOS GOBIERNOS LOCALES DE TACNA.
}

\section{RISK MANAGEMENT MODEL TO IMPROVE THE EXECUTION OF SANITATION WORKS IN TACNA'S LOCAL GOVERNMENTS}

Janeth Katherine Jinez Condori ${ }^{1}$ janethiinez93@gmail.com

Jose Antonio Salgado Canal 2

ORCID: https://orcid.org/0000-0002-5298-0704

jsalgadoc@uni.edu.pe

Aceptado: $17 / 02 / 2021$ Publicado online:15/07/2021

\section{RESUMEN}

Se realizó un estudio para diseñar un modelo de gestión de riesgos y reducir la probabilidad e impacto de los riesgos negativos y aumentar la probabilidad e impacto de los riesgos positivos, mejorando así la ejecución de obras de saneamiento en los gobiernos locales de Tacna, 2016 - 2019. Para ello, la investigación desarrollada obedece al tipo aplicada en la modalidad de innovación, porque está orientada a determinar la viabilidad del modelo de gestión de riesgos propuesto, que servi rá para mejorar la ejecución de obras de saneamiento que son ejecutadas en los gobiernos locales de la región Tacna bajo la modalidad de a dministración indirecta (contrata). Los resultados alcanzados constan del modelo de gestión de riesgos compuesto por seis procesos interrelacionados entre sí, los cuales son: planificar la gestión de ri esgos, identificar l os riesgos, análisis cualitativo de los riesgos, planificar la respuesta a los riesgos, implementar la respuesta a los riesgos y monitoreo de riesgos. Entre sus conclusiones, el modelo de gestión de ries gos compuesto por seis procesos, reduce la probabilidad e impacto de los riesgos nega tivos (amenazas); y a umenta la probabilidad e impacto de los ri esgos positivos (oportunidades); puesto que al realizarse una adecuada planificación, permitirá actuar de manera preventiva para optimizar la respuesta e incluso evitar las amenazas, minimizando sus efectos o impactos negativos durante la implementación de las respuestas; coadyuvando así a optimizar las posibilida des de éxi to, traducido en una mejora en la ejecución de obras de saneamiento en los gobi ernos locales de Tacna.

Palabras Clave: Riesgo, proceso, modelo de gestión, obra de saneamiento, obra paralizada

\section{ABSTRACT}

The present research work aims to design a risk management model to reduce the probability and impact of negative risks and increase the probability and impact of positive risks, thus improving the execution of sanitation works in the local

\footnotetext{
1 Universidad Privada de Tacna:

2 Universidad Nacional de Ingeniería
} 
governments of Tacna, 2016 - 2019. For this, the research developed obeys the type a pplied in the innovation modality, because it is a imed at determining the viability of the proposed risk management model, which wills erve to improve the exe cution of sa nitation works that a re executed in the local governments of the Ta cna region under the modality of indirect administration (contracts). The results achieved consist of the risk management model composed of six interrelated processes, which a re: planning risk ma nagement, i dentifying risks, qualitative analysis of risks, planning the response to risks, implementing the response to risks and risk monitoring. Among its conclusions we have that the risk management model composed of six processes, reduces the probability and impact of negative risks (threats); a nd increases the probability a nd impact of positive risks (opportunities); since proper planning will allow to act preventively to optimize the response and even avoid threats, minimizing their negative effects or impacts during the implementation of responses; Thus helping to optimize the chances of success, translated into an improvement in the execution of sanitation works in the local governments of Tacna.

Keywords: Risk, process, management model, sanitation, paralyzed work.

\section{INTRODUCCIÓN}

En el Perú, las obras de saneamiento ejecutadas enfrentan un alarmante problema, la gran mayoría de ellos quedan paralizadas, no llegando a cumplir con la finalidad bajo la cual fue concebida, generando que la población no cuente con infraestructura de saneamiento adecuada, tanto para agua potable como para alcantarillado, especialmente en las zonas rurales. Las obras de saneamientoque quedan sin concluir obedecen a causas diversas como por eje mplo las deficiencias en el diseño del contrato, el diseño de los expedientes técnicos y en un menor número por problemas climáticos o sociales, entre otros; ello a consecuencia que durante la ejecución de dichas obras se presentan riesgos, los cuales al no contar o tener una deficiente gestión terminan materializándose, perjudicando así a los objetivos y metas del proyecto. Asimismo, las obras de saneamiento que son eje cutadas en las zonas rurales, están mayormente a cargo de los gobiernos locales; a raíz de ello es que la presente investigación se realizó teniendo como objetivo principal diseñar un modelo de gestión de riesgos viable para mejorar la ejecución de obras de saneamiento en los gobiernos locales de Tacna; y como objetivos específicos: evaluar el estado situacional de la gestión de riesgos en gobiernos locales de Tacna durante la ejecución de proyectos de saneamiento, diseñar los procesos del modelo de gestión de riesgos y validar el modelo degestión de riesgos para mejorar la ejecución de obras de saneamiento en los gobiernos locales de Tacna.

Con la finalidad de cumplir con dichos objetivos se propone un modelo de gestión de riesgos para las obras de saneamiento ejecutadas bajo la modalidad de administración indirecta (contrata), conformado por seis procesos, los cuales son: planificar la gestión de riesgos, identificar los riesgos, análisis cualitativo de los riesgos, planificar la respuesta a los riesgos, implementar la respuesta a los riesgos y monitoreo de riesgos; los cuales se encuentran distribuidos en las etapas de planeamiento, ejecución y control; y cinco de ellas son secuenciales y uno es transversal, permitiendo así una retroalimentación continua y secuencial de los procesos del modelo. Para ello, se tuvocomo base otras investigaciones desarrolladas, de las cuales las más relevantes son tres tesis internacionales y cuatro tesis nacionales.

En el Perú, según el Plan Nacional de Saneamiento 2017-2021, 3.4 y 8.3 millones de peruanos no tienen acceso a los servicios de agua potable y alcantarillado, en los ámbitos urbano y rural, respectivamente. Asimismo, el acceso adecuado a los servicios de saneamiento impacta directamente en la calidad de vida de las personas, contribuye a mejorar la autoestima y su inclusión en la sociedad, mejora las condiciones de competitividad, disminuye la incidencia de enfermedades de origen hídrico. 
La falta de servicios de saneamiento restringe las posibilidades de las personas para llevar a cabo actividades generadoras de ingresos, conformando así el llamado círculo perverso del agua - salud pobreza. Asimismo, las inversiones realizadas en el sector saneamiento, no han logrado cerrar las brechas de acceso ni han generado impacto en la satisfacción de la población, debido a que estas no son eficientes porque los expedientes técnicos no se han formulado con criterios uniformes, no se utilizan modelos estandarizados de los componentes de infraestructura sanitaria y por los inconvenientes en la ejecución. Al cierre del año 2016, en el ámbito urbano, el Programa Nacional de Saneamiento Urbano, a nivel nacional ha identificado 121 proyectos paralizados con un presupuesto total de 933 millones de soles; a consecuencia de deficiencias en la elaboración de expedientes técnicos por falta de disponibilidad de fuente de agua, y/o de terreno, y por la mala gestión de los contratos que conllevan la demora o paralización de las obras.

\section{Figura 1}

Fotografías de las obras de saneamiento que presentaron riesgos en su ejecución:

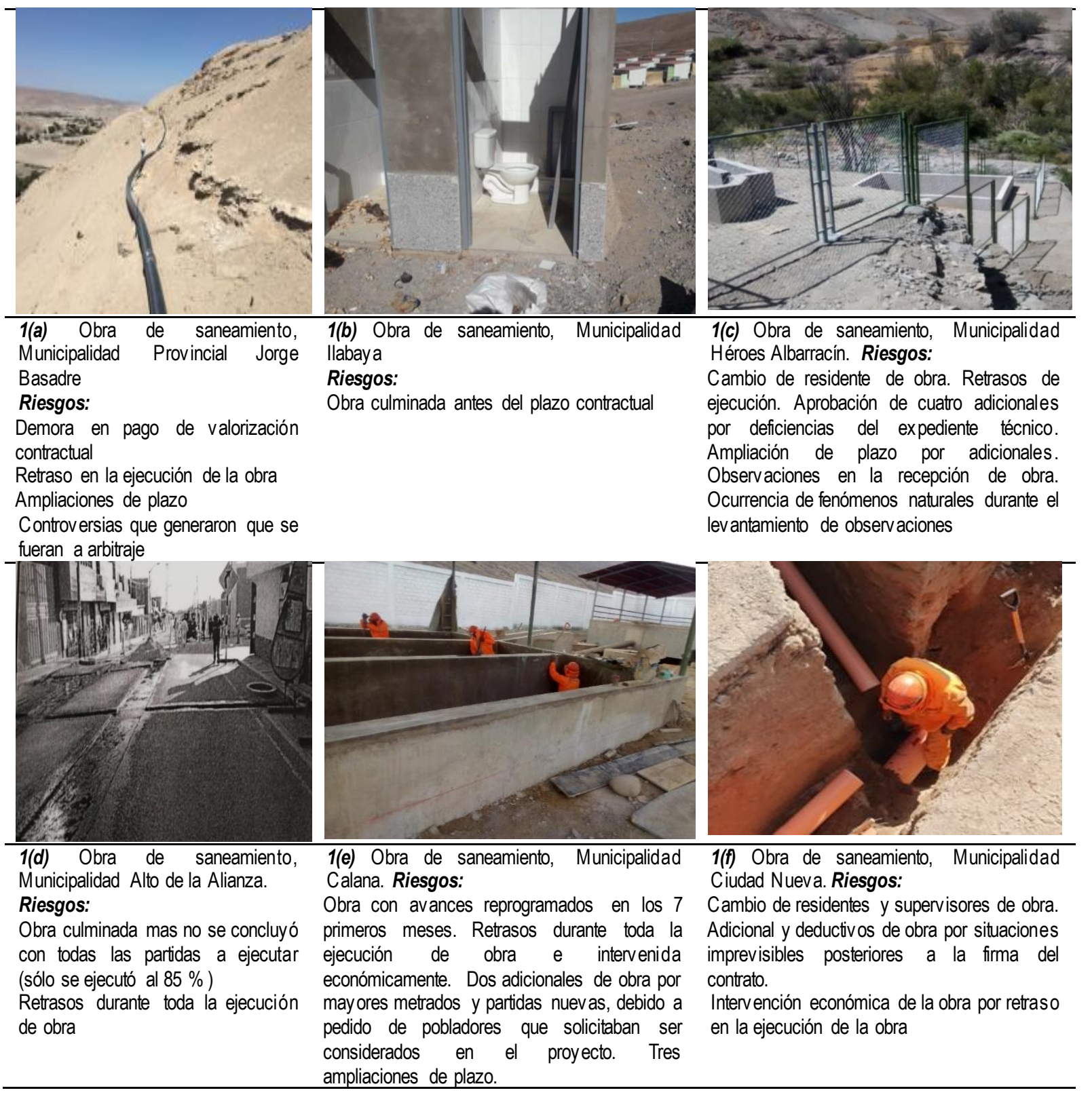


Asimismo, en el ámbito rural, el Programa Nacional de Saneamiento Rural registró 59 obras paralizadas a nivel nacional. Por su parte las municipalidades (gobiernos locales) presentan 46 obras paralizadas, cuyas causas están relacionadas a deficiencias en el diseño del contrato (resolución, suspensión o ampliación), el diseño de los expedientes técnicos y en un menor número por problemas climáticos o sociales. A ello, se suman las debilidades enel proceso constructivo de las obras, que traen como consecuencia dificultades en su o peración, lo que refleja la escasa preocupación para garantizar la sostenibilidad de las inversiones. Por otro lado, la Contraloría General de la República al 2016 identificó 201 obras de saneamiento paralizadas a nivel nacional registradas en el Sistema de Información de obras públicas "INFOBRAS" de los cuales el 75\% corresponden a obras ejecutadas por entidades del gobierno local, $14 \%$ son del gobierno regional y un $11 \%$ del gobierno nacional, siendo tal cifra reducida a 123 obras paralizadas en el año 2019. De ello, la región Tacna se ubicaba en el noveno lugar a nivel nacional, con 21 obras paralizadas. Al respecto, la Contraloría General de la República ha expuesto en su Reporte de obras paralizadas 2019.

Los factores mostrados, son los riesgos de un proyecto que han sido materializados perjudicando la ejecución de las obras, hecho que denota un inadecuado nivel de manejo de dichos riesgos, tanto en su prevención, identificacióny respuesta; es decir la mayoría de las obras paralizadas fueron a causa de la inadecuada y casi nula gestión de riesgos efectuada por los gobiernos regionales y locales. A ello se le suma que, para el caso de los gobiernos locales de Tacna, estos no conocen lo esencial de una gestión de riesgos en obras, y peor aún en los gobiernos locales de las zonas rurales. A nivel de los gobiernos locales de Tacna, de acuerdo a la información registrada en el sistema INFOBRAS de la Contraloría General de la República, se tiene que en el periodo de 2016 a 2019 se han ejecutado nueve obras de saneamiento bajo la modalidad de contrata, de las cuales ocho han presentado riesgos que no fueron gestionados. De lo descrito, se puede concluir que la ejecución de una obra se ve impactada negativamente al no tener una adecuada gestión de riesgos, permitiendo que estos se materialicen generando paralizacionese incumplimientosque no permiten que se logren los objetivos propuestos.

El riesgo de un proyecto es un evento o condición incierta que, de producirse, tiene un efecto positivo o negativo en uno o más de los objetivos del proyecto; por tanto, es fundamental la identificacióny gestión adecuada de los riesgos que representan los diversos aspectos de un proyecto. (Villalta, 2018). Es sabido que cada proyecto tiene sus particularidades y por tanto sus pro pios riesgos, sin embargo, las investigaciones existentes también señalan que la existencia de repositorios de riesgos, validados por su aplicación práctica son de utilidad para futuros proyectos. (Villalta, 2018). Por lo tanto, desde el punto de vista de la gerencia de la construcción, al contar con un adecuado modelo de gestión de riesgos validado por expertos, le permite a la entidado institución, así como al contratista contar con las herramientas necesarias para planificar, identificar, analizar e imp lementar la respuesta de forma organizada a los riesgos que se presenten durante la ejecución de alguna obra, así como realizar el monitoreo a los mismos, ello con el fin de que se mejore la ejecución de las obras de saneamiento disminuyendo la aparición de nuevos riesgos, la reincidencia en erroresy materialización de los riesgos en futuros proyectos, disminuyendo la posibilidad que terminan en paralizaciones de la obra. Asimismo, si analizamos los beneficios por el lado económico, los beneficiarios de la presente investigación son losgobiernos locales que ejecutan obras de saneamiento; ya que, al aplicar la gestión de riesgos en sus proyectos, permitirán alcanzar sus metas propuestas, mejorando la ejecución de la obra al reducir la cantidad de problemas que se presenten durante la ejecución, lo cual impactaría en los costos incurridos en el mismo.

Por otro lado, desde el punto de vista social, la población en general también se beneficiaría, debido a que, como consecuencia de la mejora en la gestión del proyecto, se podría reducir el número de obras paralizadas, contando con las obras concluidas a tiempo y pudiendo acceder a ellos oportunamente. Para ello, se debe entender quela única forma de gestionar el riesgo de manera eficaz es mediante el compromiso real de la institución o entidad, contando con una participación activa de las áreas que generan los insumos para el análisis. Los Municipios de las zonas rurales del país, han tenido un bajo desarrollo o ninguno en los análisis de gestión de riesgo, por lo que ahora deben 
destinar el recurso necesario para educar al personal sobre los beneficios en que una adecuada gestión puede resultar (Exebio, 2016).

Para Rudas Tayo, L. (2017) Incluir un modelo de gestión de riesgos en la estructura de gestión de proyectos puede transformar a cada empresa en beneficios presentes y futuros, de forma que pueda responder proactivamente a los riesgos sin incidencias que perjudiquen los intereses de la empresa o activen eventos desfavorables, y eviten Efectos improvisad os. De esta forma, se pueden lograr resultados positivos en los objetivos básicos del proyecto (tiempo, costo y calidad). Cando Ochoa, P. (2016) quien analizó la planificación integral de gestión de riesgos para los proyectos: Minicentral Hidroeléctrica y Biomasa - Aceite Piñon, dando énfasis al análisis cuantitativo a través de la elaboración de la viabilidad financiera, concluyó que la planificación integral de la gestión de riesgos de los proyectos comprende la planificación, identificación, análisis cualitativo, análisis cuantitativo, plan de respuesta y monitoreo y control. Narváez Rosero, M. (2014) presentó un modelo de gestión de riesgos para proyectos de infraestructura, aplicable para construcción de edificios en la fase de diseño. Para ello empleó la metodología propuesta por la Guía PMBOK que contiene la identificación, análisis cualitativo y cuantitativo, y los planes de contingencia y observó que los riesgos del tipo operacional y técnico son los más incidentes a dichos proyectos. Quevedo Porras, V. (2019), quien demostró que en la Región de Tacna la elaboración y empleo de la Gestión de Riesgos en la ejecución de proyectos de saneamiento es bajo. Llegó a la conclusión de una necesidad que todos los funcionarios, ejecutivos, profesionales y personas involucradas con la dirección y gestión de los proyectos tengan las competencias profesionales que les permita priorizar la gestión de riesgos para mejorar el alcance y lograr que los costos y tiempos se acerquen al valor planeado. El éxito de un proyecto se logra cuando se comprende los riesgos que se enfrenta y adopta procesos de gestión. Saloma Valdivia, D. (2018), logró demostrar que la Hipótesis Principal que dice: "La aplicación de una adecuado modelo para la gestión de riesgos permitirá mejorar la ejecución de Intercambios Viales Subterráneos con el método constructivo cut and cover - top down, el cual dependerá del análisis de la identificación de los riesgos, rentabilidad económica y desarrollo sostenible"; se sustenta con los procedimientos planteados para la gestión de los riesgos, plasmados en los procesos para la gestión de riesgos, procedimiento para identificación de los riesgos, análisis de riesgos y asignación de riesgos. Este modelo permitirá hacer la evaluación estadística de la afectación en plazo y costo para la ejecución del proyecto, lo cual ha sido demostrado a través de la Simulación Monte Carlo realizada para el IVD Benavides. El modelo de gestión de riesgos propuesto ha sido elaborado teniendo como consideración específica la ejecución de intercambios viales subterráneos que utilizan el método constructivocut and cover - top down; sin embargo, los procesos, procedimientos y tareas identificadas permite que esta metodología sea válida para ser utilizada para la ejecución de intercambios viales subterráneos, teniendo en consideraciones las variaciones respectivas. Villalta Paredes, C. (2018), ha identificado los riesgos en la gestión contractual de proyectos públicos de infraestructura educativa, tomando como referencia fundamentalmente la estructura de desglose de riesgos de la Guía del PMBOK del PMl en sus ediciones quinta y sexta, correspondientes a los años 2013 y 2017. Producto de ello se han identificado 4 categorías de riesgo: técnico, de gestión, comercial y externo, para las cuales se han establecido 30 diferentes fuentes de riesgo, y para cada una de ellas un listado de uno o más riesgos que en conjunto hacen un total de 115 riesgos específicos aplicables a la gestión contractual de proyectos públicos de infraestructura educativa. Tello, Barboza y Rodríguez (2016), luego de la evaluación de proyectos ejecutados y la información recabada de los diferentes agentes encargados de la ejecución de los proyectos, afirma que ante las dificultades que se presentan duranteel proceso de ejecución de obra todas las decisiones de solución son reactivas, no contándose con un plan de gestión de riesgos que ayuden a tomar las mejores decisiones ante situaciones de incertidumbre, los impactos en los proyectos se muestran que son mayores que si se hubiera tenido un plande respuesta.

"Los riesgos en un proyecto de construcción e stán presentes en todas las etapas de su ejecución y muchas veces des de su elaboración. Estos riesgos se involucran directamente con las áreas o equipos de trabajo encargadas del proyecto." (Hurtado, 2019). Kliem \& Ludin (citado en Malpartida, 2018) indican que "el riesgo es simplemente la posibilidad de 
ocurrencia de un evento que pudiera tener consecuencias o impactos en un proyecto, ya sean éstos positivos o negativos. Además, añade que los elementos del riesgo que deben ser considerados por los gerentes de proyectos son: La probabilidad de ocurrencia del riesgo, la frecuencia o tiempo de retorno del evento de riesgo, el impacto que podría generar en caso de ocurrir, la importancia relativa a otros riesgos y la vulnerabilidad o grado de exposición, que es producto del impacto y la probabilidad".

Por ello, al querer desarrollar un modelo de gestión de riesgos, se de be considerar obligatoriamente lo establecido en la normativa peruana, siendo para el presente caso lo e stablecido en la Directiva N012-2017-OSCE/CD “Gestión de riesgos en la planificación de la ejecución de obras", elaborado por el Organismo Supervisor de las Contrataciones del Estado (OSCE) el 23 de mayo de 2017, que desarrolla 4 procesos: identificar riesgos, analizar riesgos, planificar la respuesta a riesgos y asignar riesgos; así como los lineamientos desarrollados en la Guía de los fundamentos para la dirección de proyectos (PMBOK), cuya aplicación no es obligatoria, pero si nos proporciona buenas prácticas respecto a los siguientes procesos de la gestión de riesgos de un proyecto: la planificación, identificación, análisis cualitativo y cuantitativo, planificar e implementar la respuesta y monitorear los riesgos. Según el Plan Nacional de Saneamiento 2017-2021, en el país 3,4 y 8,3 millones de habitantes no cuentan con agua potable y alcantarillado, en los ámbitos rural y urbano (Ministerio de Vivienda Construcción y Saneamiento, 2017). Ante ello, planteó que se invierta en el desarrollo de infraestructura de producción de agua potable, ejecución de obras de saneamiento y tratamiento de aguas residuales, a fin de superar la problemática descrita. Es asíque los tres niveles de gobierno; central, regional y local, invierten recursos para la ejecución de proyectos de agua y saneamiento. Sin embargo, en varios casos reportados a la Defensoría del Pueblo, se advierten paralizaciones de obras y demoras excesivas de hasta ocho años para su culminación. (Defensoría del Pueblo, 2014). Las obras de saneamiento, según el artículo 268 del Reglamento de la Ley de Contrataciones, aprobado con el Decreto Supremo $\mathrm{N}^{\circ} 184$ 2008-EF (2008), comprenden las plantas de tratamiento de agua potable, redes de conducción de agua potable, redes de conducción de desagües, conjuntos habitacionales, habilitaciones urbanas, emisores de desagüe, líneas de impulsión, líneas de aducción, líneas de conducción, cámaras de bombeo, reservorios elevados o apoyados, lagunas de oxidación, conexiones domiciliarias de agua y desagüe, plantas de tratamiento. Asimismo, se entiende como proyecto de inversión en saneamiento a aquel que se orienta a la creación, ampliación, mejoramientomodernización o recuperación de la capacidad productora de servicios de: agua potable, alcantarillado, tratamiento de aguas servidas, disposición de excretas o desagüe pluvial. (Directiva N001-2006-EF/68.01, 2006).

\section{OBJETIVOS}

Evaluar el estado situacional de la gestión de riesgos en gobiernos locales de Tacna durante la ejecución de proyectos de saneamiento.

Diseñar los procesos del modelo de gestión de riesgos que permita mejorar la ejecución de obras de saneamiento en los gobiernos locales de Tacna.

Validar el modelode gestión de riesgos para mejorar la ejecución de obras de saneamiento en los gobiernos locales de Tacna.

\section{METODOLOGÍA}

Por la finalidad es una investigación del tipo aplicada en la modalidad de innovación en la gestión de riesgos, porque está orientada a determinar la viabilidad del modelo de ge stión de riesgos para mejorar la ejecución de obras de saneamiento en gobiernos locales de la región Tacna. Ello teniendo en cuenta que la investigación aplicada busca la generación de conocimiento con aplicación directa a los problemas de la sociedad o el sector productivo. Esta se basa fundamentalmente en los 
hallazgos tecnológicos de la investigación básica, ocupándose del proceso de enlace entre la teoría y el producto. (Lozada, 2014). Asimismo, para Murillo (2008), la investigación aplicada recibe el nombre de "investigación práctica o empírica", que se caracteriza porque busca la aplicación o utilización de los conocimientos adquiridos, a la vez que se adquieren otros, después de implementary sistematizar la práctica basada en investigación. El tipo de investigación es el propositivo. El ámbito de estudio corresponde entonces los gobiernos locales de Tacna (municipios provinciales y distritales) y el tiempo social de análisis es el período de 2016 a 2019. La población de estudio estuvo conformada por los ingenieros civiles, con experiencia mínima de 5 años, quienes han trabajado en obras de saneamiento. Al aplicar la fórmula para obtener la muestra en base a una población total de 1212 ingenieros colegiados en Tacna, lo cual representa una población grande, nos da una muestra de 89 ingenieros.

Para la validación del modelo propuesto se rá por juicio de expertos ( 6 ingenieros con amplia experiencia en gestión de riesgos y ejecución de obras, y preferentemente en obras de saneamiento y/o cargos de gerencia).

Se revisaron los documentos respecto a la ejecución de obras de saneamiento en varios gobiernos locales de Tacna, tales como informes, reportes, entre otros. Para ello se revisó la información publicada en los aplicativos del MEF: Sistema de Seguimiento de Inversiones (SSI) y Consulta amigable, lo cual nos proporciona información sobre la inversión y los gastos realizados en cada obra de saneamiento ejecutada en los gobiernos locales de Tacna; Invierte.pe y Sistema Nacional de Inversión Pública (SNIP), a fin de obtener mayor información de lo concerniente a la etapa de pre inversión de cada obra de saneamiento ejecutada; Infobras de la Contraloría General de la República, a fin de extraer datos respecto a la ejecución de la obra, tales como avances mensual es, problemas presentados, estado actual de la obra, entre otros.

Los instrumentos para la recolección de datos fueron los siguientes: a) Cuestionario: Para el diagnóstico, el cuestionario aplicado a través de una encuesta realizada a 30 profesionales, constó de veinte preguntas distribuidas en tres secciones (Datos generales del encuestado, conocimiento del problema y Pertinencia y necesidad de una propuesta de solución). Para la validación, el cuestionario aplicado a través de una entrevista realizada a seis expertos, constó de seis preguntas concernientes al grado de validez que le otorgan a cada proceso del modelo de gestión de riesgos propuesto en la presente investigación. b) Archivos documentarios. Se emplearon los documentos de la ejecución de obras de saneamiento, tales como base de datos o información histórica de las obras de saneamiento ejecutadas en los gobiernos locales de Tacna, los cuáles se encuentran publicados en los aplicativos Sistema de Seguimiento de Inversiones (SSI) y Consulta ami gable del Ministerio de Economía y Finanzas (MEF); Invierte.pe y Sistema Nacional de Inversión Pública (SNIP); Infobras de la Contraloría General de la República, entre otros. Ello, con el fin de obtener datos que permitan realizar una lista de riesgos que se presentan en la ejecución de obras de saneamiento, para luego categorizarlos y considerarlos dentro del modelo propuesto.

Para el análisis estadístico se aplicó las técnicas y procedimientos de la estadística descriptiva, que comprende la elaboración de tablas estadísticas y diseño de figuras que representan el comportamiento de las variables. Para el análisis inferencial se aplicó la distribución estadística TStudents para la validación del modelo.

\section{RESULTADOS}

En el nivel de necesidad de la propuesta de innovación, se observa que el $90 \%$ considera que hay una alta necesidad de una propuesta innovadora y un $10 \%$ considera que hay una baja necesidad de la propuesta. En tal sentido se comprueba que la mayoría de profesionales cree que es necesario una propuesta de innovación para solucionar el problema de la gestión de riesgos y refleja un alto interés con lo propuesto en el tema. 
Tabla 1

Diagnóstico, de la confiablidad general de los encuestados, nivel de conocimiento del problema y necesidad de una propuesta de solución desde la perspectiva de 30 profesionales.

\begin{tabular}{lcc}
\hline \multicolumn{1}{c}{ Nivel } & Frecuencia & Porcentaje \\
& & \\
\hline \multicolumn{1}{c}{ Confiabilidad de la información } & general & de los encuestados \\
\hline Alta confiabilidad & 28 & 93,30 \\
Baja confiabilidad & 2 & 6,70 \\
Total & $\mathbf{3 0}$ & $\mathbf{1 0 0}$ \\
\hline \multicolumn{4}{c}{ Nivel de conocimiento de la problemática } \\
\hline Alto conocimiento & 26 & 86,70 \\
Bajo conocimiento & 4 & 13,30 \\
Total & $\mathbf{3 0}$ & $\mathbf{1 0 0}$ \\
\hline \multicolumn{4}{c}{ Nivel de necesidad } & de la propuesta \\
\hline Alto nivel de necesidad de la propuesta & 27 & 90,00 \\
Bajo nivel de necesidad de la propuesta & 3 & 10,00 \\
Total & $\mathbf{3 0}$ & $\mathbf{1 0 0}$ \\
\hline
\end{tabular}

El $86.67 \%$ de profesionales considera necesario y conveniente contar con un modelo de gestión de riesgos para reducir la probabilidad de ocurrencia de los riesgos en obras. El 100\% concluye que la propuesta del modelo de gestión de riesgos debe incluir procesos para identificar, categorizar y evaluar la probabilidad e impacto de los riesgos.

\section{PROPUESTA DE MODELO DE GESTIÓN DE RIESGOS}

\section{DEFINICIÓN DE LA PROPUESTA:}

El Modelo de Gestión de Riesgos para mejorar la ejecución de obras de saneamiento, tiene por finalidad diseñar los procesos y procedimientos para gestionar los riesgos que se presentan en obras de saneamiento ejecutadas bajo la modalidad de administración indirecta (contrata) por los gobiernos locales de Tacna, 2016 - 2019.

\section{CARATERÍSTICAS DE LA PROPUESTA:}

La propuesta se caracteriza por presentar seis procesos interrelacionad os, los cuales son:

- P1: Planificar lagestión de riesgos

- P2: Identificar los riesgos

- P3: Análisis cualitativo de riesgos

- P4: Planificar la respuesta a los riesgos

- P5: Implementar la respuesta a los riesgos

- P6: Monitoreo de los riesgos

Se precisa que el modelo no considera el proceso de análisis cuantitativo de riesgos descrito en la guía PMBOK 6ta edición. 
Figura 1

Procesos del modelo de gestión de riesgos propuesto

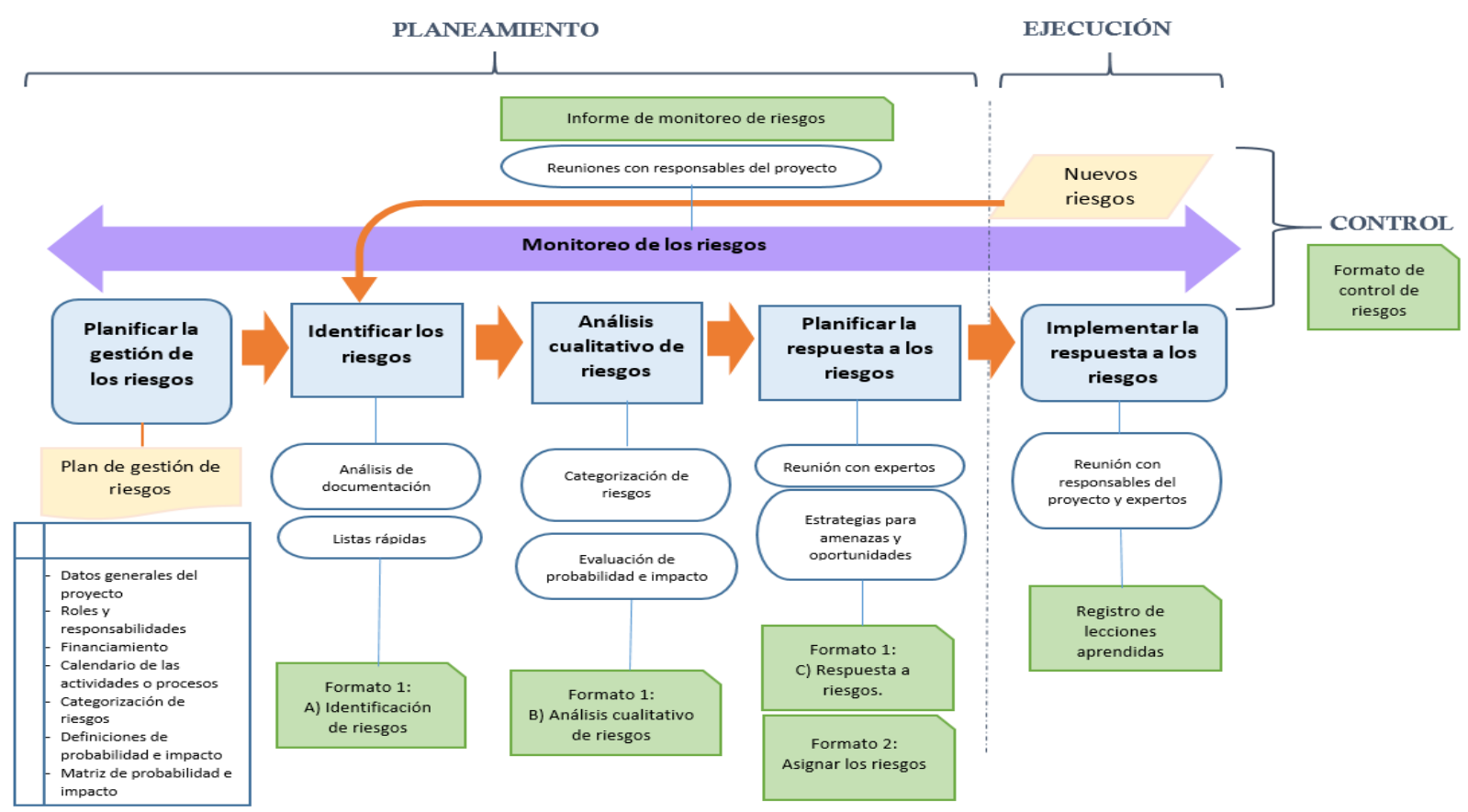

\section{PROCESO 1: PLANIFICAR LA GESTIÓN DE LOS RIESGOS}

El proceso de planificar la gestión de riesgos consiste en definir las actividades a realizar, a fin de asegurar que el nivel, tipo y la visibilidad de gestión de riesgos, son proporcionales tanto los riesgos como a la importancia del proyecto. Este proceso se lleva a cabo una única vez, excepto que posteriormente surjan condiciones en el ciclo de vida del proyecto que requieran que se vuelva a examinar este proceso, tales como: si el alcance del proyecto cambia significativamente o si en un examen posterior de la efectividad de la gestión de riesgos determina que este requiere modificaciones. A continuación, se presentan y describen las entradas, herramientas y técnicas, y salidas del proceso de planificación:

\section{PROCESO 2: IDENTIFICAR LOS RIESGOS}

El proceso de identificar los riesgos consiste en identificar los riesgos individuales del proyecto, así como las fuentes de riesgo general del proyecto y documentar sus características. Este proceso iterativo, es decir que se lleva a cabo a lo largo del proyecto, puesto que pueden aparecer nuevos riesgos del proyecto que deberán ser adecuadamente identificados.

\section{PROCESO 3: ANÁLISIS CUALITATIVODE LOS RIESGOS}

El proceso de análisis cualitativo de riesgos consiste en priorizar los riesgos individuales del proyecto evaluando la probabilidad de ocurrencia e impacto de dichos riesgos en los objetivos del proyecto.

\section{PROCESO 4: PLANIFICAR LA RESPUESTA A LOS RIESGOS}

El proceso de planificar la respuesta a los riesgos consiste en desarrollar estrategias y acordar acciones para tratar los riesgos individuales del proyecto. Este proceso se lleva a cabo a lo largo de todo el proyecto. (PMBOK, 2017)

\section{PROCESO 5: IMPLEMENTAR LA RESPUESTA A LOS RIESGOS}

El proceso de implementar la respuesta a los riesgos consiste en ejecutar las acciones de 
respuesta a los riesgos tal como se planificaron, y que se tomen las medidas para gestionar el riesgo, para que no sólo se quede en documentos. Para ello, se debe considerar que las respuestas planificadas deben ser implementadas a la brevedad posible, dependiendo de las restricciones que tenga el proyecto, una vez detectada la aparición del riesgo.

\section{PROCESO 6: MONITOREO DE LOS RIESGOS}

El proceso de monitorear los riesgos, consiste en hacer seguimiento a los riesgosidentificados, identificary analizar los nuevos riesgos y evaluar la efectividad del proceso de gestión de los riesgos. Siendo así, este proceso se desarrolla a lo largo de todo el proyecto, con la intención de determinar si: las respuestas a los riesgos implementadas son efectivas, el estado de los riesgos individuales del proyecto ha cambiado, han aparecido nuevos riesgos individuales del proyecto, se respetan las políticas y procedimientos de gestión de riesgos y si las estrategias de gestión siguen siendo válidas.

\section{DESCRIPCIÓN DE LOS RESULTADOS DE LA PROPUESTA}

Para la validación de la propuesta se realizó una segunda encuesta, pero sólo a un grupo de expertos, y se diseñó un formato tipo, de donde se obtuvo la media de las respuestas brindadas por los seis expertos entrevistados, según se muestra en la siguiente tabla:

Tabla 2

Resultados de la aplicación de los procesos del modelo propuesto

\begin{tabular}{lcc}
\hline \multicolumn{1}{c}{ Dimensión del modelo } & Media & Grado de validez \\
\hline Componente 1: Planeamiento & & \\
\hline Proceso 1: Planificar la gestión de riesgos & 3.00 & Alto \\
\hline Proceso 2: Identificar los riesgos & 3.00 & Alto \\
\hline Proceso 3: Análisis cualitativo de riesgos & 2.67 & Al to \\
\hline Proceso 4: Planificar la respuesta a los riesgos & 2.67 & Alto \\
\hline Componente 2 y 3: Ejecución y control & & \\
\hline Proceso 5: Implementar la respuesta a los riesgos & 2.50 & Medio \\
\hline Proceso 6: Monitoreo de riesgos & 2.50 & Medio \\
\hline
\end{tabular}

De la tabla precedente se aprecia que los últimos dos procesos de implementación de la respuesta a los riesgos y el monitoreo de riesgos obtuvo un nivel de aceptación “Medio", ante ello se formulan las siguientes recomendaciones a tener en cuenta al aplicar el modelo de gestión de riesgos propuesto:

\section{Proceso 5: Implementar la respuesta a los riesgos}

Considerar que durante la ejecución de la obra las reuniones que se desarrollaran con los responsables del proyecto, se deben realizar periódicamente, y registrando todo lo acontecido respecto a los riesgos que se presentaron y los que están probables a presentarse en el formato de Acta de reuniones, donde además al identificar un nuevo riesgo que no estuvo considerado en la planificación inicial, se debereal izar todo el proceso de gestión de riesgos para ellos; procediendo a la actualización de los documentos de la gestión de riesgos

\section{Proceso 6: Monitoreo de riesgos}

Se recomienda que al realizar el monitoreo de los riesgos, se cuente con la presencia de presencia de expertos o se debe contratar una oficina especializada de expertos que permita evaluar cómo se da el desarrollo de la gestión de riesgos y si las estrategias que se están aplicando están resultando las pertinentes; asimismo que estos se encarguen de realizar auditorías externas que permitan develar el estado, la eficiencia y el cumplimiento de la gestión de riesgos que se está aplicando en el proyecto. 
Se concluye que, con un nivel de confianza del $95 \%$ el nivel de validez del modelo de gestión de riesgos propuesto es alta; por lo tanto, constituye una alternativa viable para la solución del problema de investigación, según los expertos $(p<0.00)$.

\section{CONCLUSIONES}

Se diseñó un Modelo de Gestión de Riesgos compuesto por seis procesos que reduce la probabilidad e impacto de los riesgos negativos (amenazas), tales como: expropiaciones, desabastecimiento de materiales, presencia de restos arqueológicos, roturas de redes existentes, adicionales de obra, ampliaciones de plazo, documentación técnica deficiente, entre otros; y aumenta la probabilidad e impacto de los riesgos positivos (oportunidades), tales como: experiencia y capacidades del contratista, personal obrero muy calificado, nuevas tecnologías, entre otros; puesto que al realizarse una adecuada planificación, permitirá actuar de manera preventiva para optimizar la respuesta e incluso evitar las amenazas, minimizando sus efectos o impactos negativos durante la implementación de las respuestas; coadyuvando así a optimizar las posibilidades de éxito, traducido en una mejora en la ejecución de obras de saneamiento en los gobiernos locales de Tacna. Se recomienda a los gobiernos locales de Tacna, que adopten un Modelo de Gestión de Riesgos para la ejecución de obras de saneamiento, tomando en consideración el modelo propuesto y los formatos diseñados.

Se evaluó el estado situacional de la gestión de riesgos en gobiernos locales de Tacna durante la ejecución de proyectos de saneamiento, a través de la aplicación de un cuestionario a 30 profesionales, de los cuales el $66.67 \%$ señaló que la gestión de riesgos en las obras de saneamiento es poca o casi nula; asimismo, y a consecuencia de ello, el $66.67 \%$ de encuestados reveló que durante la ejecución de obras de saneamiento se han presentado y materializado diversos riesgos, con lo cual se demuestra que se tiene una deficiente gestión de riesgos, haciendo necesario un nuevo modelo de gestión de riesgos innovador. Asimismo, del análisis realizado a la ejecución de las obras de saneamiento por contrata del 2016 al 2019, se ha evidenciado que se presentaron o materializaron diversos riesgos que retrasaron e incluso no permitieron la culminación de dichas obras, tales como: demora en pago de valorizaciones, ampliaciones de plazo, cambios continuos de residente de obra, fenómenos naturales, intervenciones económicas, adicionales de obra, entre otros. Se recomienda al área de Recursos Humanos de cada gobierno local de Tacna, que programen y gestionen capacitaciones relacionadas a la gestión de riesgos en proyectos para sus funcionarios y profesionales que intervengan durante la gestión del proyecto, tales como oficina de estudios, área logística, oficina de planeamiento, área o gerencia de ejecución de obras, área de supervisión de obras, entre otros de acuerdo a la realidad de cada gobierno local.

Se diseñó un modelo de gestión de riesgos con los procesos y procedimientos para gestionar los riesgos que se presentan en obras de saneamiento ejecutadas bajo la modalidad de administración indirecta (contrata), por los gobiernos locales de Tacna, el cual corresponde a una innovación a través de la actualización de la Directiva N 012-2017-OSCE/CD “Gestión de riesgos en la planificación de la ejecución de obras", agregando los procesos de implementación, monitoreo y control basados en lo descrito en la guía del PMBOK 6ta edición; quedando finalmente compuesta por seis procesos: planificar la gestión de riesgos, identificar los riesgos, análisis cualitativo de los riesgos, planificar la respuesta a los riesgos, implementar la respuesta a los riesgos y moni toreo de riesgos, los cuales se encuentran relacionados entre sí de manera secuencial y transversal, de tal forma que su aplicación se realiza de manera integral. Se recomienda a los gobiernos locales de Tacna que consideren la participación de un experto o grupo de expertos que facilite la adopción de la gestión de riesgos y oriente en la aplicación de cada proceso, lo que permitirá disminuir la probabilidad de optar por una planificación e implementación de la gestión de riesgos inadecuada.

El modelo de gestión de riesgos propuesto fue validado por seis expertos, otorgándole grados de validez alta y media a cada uno de los procesos; determinando así que el modelo de gestión de 
riesgos propuesto constituye una alternativa viable para mejorar la ejecución de obras de saneamiento en gobiernos locales de la región Tacna, con un nivel de confianza del 95\%. Se recomienda que, de considerarlo pertinente, y luego de evaluada y aprobada la presente tesis por los jurados correspondientes, la Escuela de Postgrado pueda hacer llegar el modelo propuesto a los gobiemos locales de Tacna para su implementación. Se recomienda a las universidades públicas y privadas de Tacna, implementar o dictar un curso para las carreras de ingeniería y arquitectura, que contemple e ilustre sobre la gestión de riesgos en proyectos u obras.

\section{REFERENCIAS}

Cando Ochoa, Paola S. (2016). Modelo de gestión de riesgos en proyectos de inversión de la Subsecretaría de Energía Renovable del Ministerio de Electricidad y Energía Renovable. Universidad Andina Simón Bolívar, Quito.

Contraloría General de la República (2019). Reporte de obras paralizadas 2019. Sitio web de la contraloría:

http://doc.contraloria.gob.pe/estudios-especiales/documento trabajo/2019/

Reporte_Obras_Paralizadas.pdf

Decreto Supremo N 184-2008-EF (2008). Reglamento de la Ley de Contrataciones del Estado. Perú, 1 de enero de 2009.

Decreto Supremo N 019-2017-VIVIENDA (2017). Reglamento de la Ley Marco de la Gestión y prestación de los servicios de saneamiento. Perú, 26 de junio de 2017.

Defensoría del Pueblo (2014). Cumplimiento y vigilancia de la ejecución de los proyectos de agua y saneamiento. Sitio web de la defensoría:

https://www.defensoria.gob.pe/blog/cumplimiento-y-vigilancia-de-la-ejecucion-de-losproyectos-de-agua-y-saneamiento/

Del Rincón Igea, Delio (1995). Técnicas de investigación en ciencias sociales. Dykinson.

Directiva $\mathrm{N}^{\circ}$ 001-2006-EF/68.01 (2006). Directiva para proyectos de inversión en saneamiento formulados y ejecutados porterceros. Perú, 15 de junio de 2006.

Directiva N012-2017-OSCE/CD. Gestión de Riesgos en la Planificación de la Ejecución de obras. Perú, 23 de mayo de 2017.

Exebio Lozano, Carol G. (2016). Plan de Gestión de riesgos para la obra del sistema de agua potablee instalación de letrinas en el caserio de Sayapampa distrito de Curgos - Sanchez Carrion - La Libertad. Universidad Privada Antenor Orrego, Trujillo.

Hurtado Zamora, Victor (2019). Propuesta para la gestión de riesgos en la obra Mejoramiento del servicio de transitabilidad vial de la prolongación calle Francisco de Zela, de la ciudad de Trujillo. Universidad Privada Antenor Orrego, Trujillo.

Lozada, José (2014). Investigación A plicada Definición, Propiedad Intelectual e Industria. Revista digital "CienciAmérica: Revista de divulgación científica de la Universidad Tecnológica Indoamérica", volumen 3, págs. 47-50. https://dialnet.unirioja.es/servlet/articulo?codigo=6163749.

Malpartida Livia, Kevin Jhordy (2018). Aplicación de gestión de riesgos en la ejecución de proyectos de edificación en la provincia de Pasco - 2018. Universidad Nacional Daniel Alcides Carrión.

Murillo Hernandez, Willian J. (2008). La investigación científica. Sitio web de monografías: https://www.monografias.com/trabajos15/invest-cientifica/invest-cientifica.shtml

Narváez Rosero, María del Pilar (2014). Gestión de riesgos en la fase de diseño para proyectos de construcción utilizando la guía PMBOK. Artículo para la Universidad Militar Nueva Granada.

Project Management Institute (2017). Guía de los fundamentos para la dirección de proyectos. Lima.

Quevedo Porras, Violeta (2017). Modelo de gestión de riesgos y su impacto en el alcance, tiempo y costo de los proyectos de saneamiento básico en la región Tacna, 2017. Universidad Privada de Tacna.

Rudas Tayo, Leidy P. (2017). Modelo de gestión de riesgos para proyectos de desarrollo tecnológico. Ciateq. 
Salgado Canal, Jose A. (2010). Importancia de la planificación, para el éxito de los proyectos, aplicando una metodología estándar de gestión de proyectos. Universidad Nacional de Ingeniería.

Saloma Valdivia, Diego E. (2018). Modelo de gestión de riesgos para mejorar la ejecución de intercambios viales subterráneos que utilizan el método constructivo Cut And Cover - Top. Universidad Nacional de Ingeniería.

Tello, Barboza y Rodríguez (2016), Propuesta de gestión de riesgos en proyectos de inversión pública en la Municipalidad Distrital de Baños del Inca, aplicando la metodología del PMI orientada a la sistematización de riesgos en el año 2016. Universidad Privada Antonio Guillermo Urrelo, Cajamarca.

Villalta Paredes, Cesar O. (2018). Gestión de riesgos en la ejecución contractualde proyectos públicos de infraestructura educativa en colegios emblemáticos de la ciudad de Arequipa. Universidad Nacional de San Agustín. 